\title{
Neurodegeneration by polyglutamine Atrophin is not rescued by induction of autophagy
}

\author{
I Nisoli ${ }^{1}$, JP Chauvin ${ }^{2}$, F Napoletano ${ }^{1}$, P Calamita ${ }^{1}$, V Zanin ${ }^{1}$, M Fanto ${ }^{\star, 1,3,4}$ and B Charroux $x^{\star, 2,4}$
}

Polyglutamine pathologies are neurodegenerative diseases that manifest both general polyglutamine toxicity and mutant protein-specific effects. Dentatorubral-pallidoluysian Atrophy (DRPLA) is one of these disorders caused by mutations in the Atrophin-1 protein. We have generated several models for DRPLA in Drosophila and analysed the mechanisms of cellular and organism toxicity. Our genetic and ultrastructural analysis of neurodegeneration suggests that autophagy may have a role in cellular degeneration when polyglutamine proteins are overexpressed in neuronal and glial cells. Clearance of autophagic organelles is blocked at the lysosomal level after correct fusion between autophagosomes and lysosomes. This leads to accumulation of autofluorescent pigments and proteinaceous residues usually degraded by the autophagy-lysosome system. Under these circumstances, further pharmacological and genetic induction of autophagy does not rescue neurodegeneration by polyglutamine Atrophins, in contrast to many other neurodegenerative conditions. Our data thus provide a crucial insight into the specific mechanism of a polyglutamine disease and reveal important differences in the role of autophagy with respect to other diseases of the same family.

Cell Death and Differentiation (2010) 17, 1577-1587; doi:10.1038/cdd.2010.31; published online 26 March 2010

Polyglutamine (polyQ) pathologies are a family of dominantly inherited neurodegenerative diseases caused by mutations in which an expanded CAG repeat tract results in a long stretch of Qs in the encoded protein. This family includes Huntington Disease, Dentatorubral-pallidoluysian Atrophy (DRPLA) and several spinocerebellar ataxias. Apart from their polyQ repeats, the proteins involved are unrelated, and although they are all widely expressed in the CNS and peripheral tissues, they lead to distinct patterns of neurodegeneration. ${ }^{1}$ PolyQ-expanded proteins misfold and accumulate in large aggregates, which have been initially described as toxic, ${ }^{1}$ and more recently as a positive prognosis factor in neuronal survival. $^{2}$ The molecular and cellular mechanisms of toxicity due to polyQ proteins are not yet fully characterised and many of the principal aspects are under intense scrutiny. In particular, many reports have described autophagy as a protective cellular mechanism in neurodegeneration, ${ }^{3}$ although its full contribution to the pathogenesis, including cell killing, is still poorly understood. The full cycle of autophagic degradation of cellular components and recycle of simpler constituents is not involved in cell killing. However, many dysfunctions at different steps of this cycle that have been reported upon expression of toxic proteins, including polyQ proteins, can lead to cell degeneration and death. ${ }^{4}$

Over the past few years a growing number of studies have focused on identifying the interplay between polyQ effects and protein-specific misfunctions, ${ }^{5-7}$ with the assumption that
polyQ pathologies combine general polyQ toxicity with disease-specific effects due to the proteins affected. The fruitfly Drosophila melanogaster has proved to be a valuable model organism for polyQ diseases and neurodegeneration. ${ }^{8}$ New models have also moved on from initial basic observations, uncovering the complex interplay between polyQ effects and RNA or protein-specific misfunction. 5,7,9

We generated several Drosophila models of DRPLA (Figure 1a and Supplementary Figure 1), a polyQ disease caused by mutations in atrophin-1. ${ }^{10,11}$ Atrophins are transcriptional cofactors conserved from Drosophila to mammals, ${ }^{12-15}$ providing an ideal background for the dissection of polyQ effects and specific Atrophin functions through Drosophila genetics. We show that polyQ Atrophins promote neurodegeneration with autophagic hallmarks both in neuronal photoreceptors and glial cells. Through blocking lysosomal digestion, they hamper the beneficial effects of further autophagy induction in contrast to other proteinopathies. Thus, our data reveal a specific mechanism of toxicity of a polyglutamine disease and uncover a complex interplay of Atrophins with autophagy.

\section{Results}

Retinal degeneration by Drosophila Atro and human Atrophin-1. The Drosophila Atrophin gene (Atro) encodes a large ubiquitous protein containing all the functional domains of Atrophins, including two polyQ stretches. ${ }^{12,14}$ We have

\footnotetext{
${ }^{1}$ Dulbecco Telethon Institute and Division of Neuroscience, DIBIT-San Raffaele Scientific Institute, Via Olgettina 58, Milan I-20132, Italy; ${ }^{2}$ Institut de Biologie du Développement de Marseille Luminy (IBDML), UMR 6216, Campus de Luminy Case 907, Marseille Cedex 9 F-13288, France and ${ }^{3}$ MRC Centre For Developmental Neurobiology, King's College London, Guy's Campus, London SE1 1UL, UK

${ }^{*}$ Corresponding authors: M Fanto, MRC Centre For Developmental Neurobiology, King's College London, New Hunt's House, Guy's Campus, London SE1 1UL, UK. Tel: + 4420784 86807; Fax: + 4420784 86550; E-mail: manolis.fanto @ kcl.ac.uk or B Charroux, Institut de Biologie du Développement de Marseille Luminy (IBDML), UMR 6216, Campus de Luminy, Case 907, Marseille Cedex 9 F-13288, France; Tel: +33 (0)491 269 324; E-mail: charroux@ibdml.univ-mrs.fr

${ }^{4}$ These authors contributed equally to this work.

Keywords: neurodegeneration; Drosophila; autophagy; atrophin

Abbreviations: DRPLA, Dentatorubral-pallidoluysian Atrophy; polyQ, polyglutamine

Received 14.9.09; revised 18.2.10; accepted 18.2.10; Edited by E Baehrecke; published online 26.3.10
} 

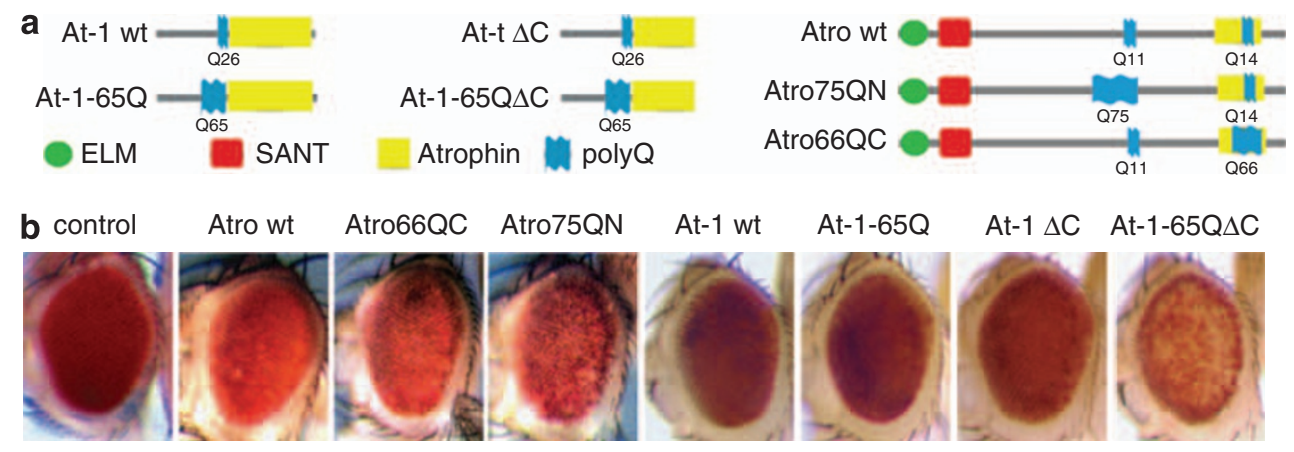

C

control

Atro wt

Atro66QC

Atro75QN

$1 \mathrm{~d}$
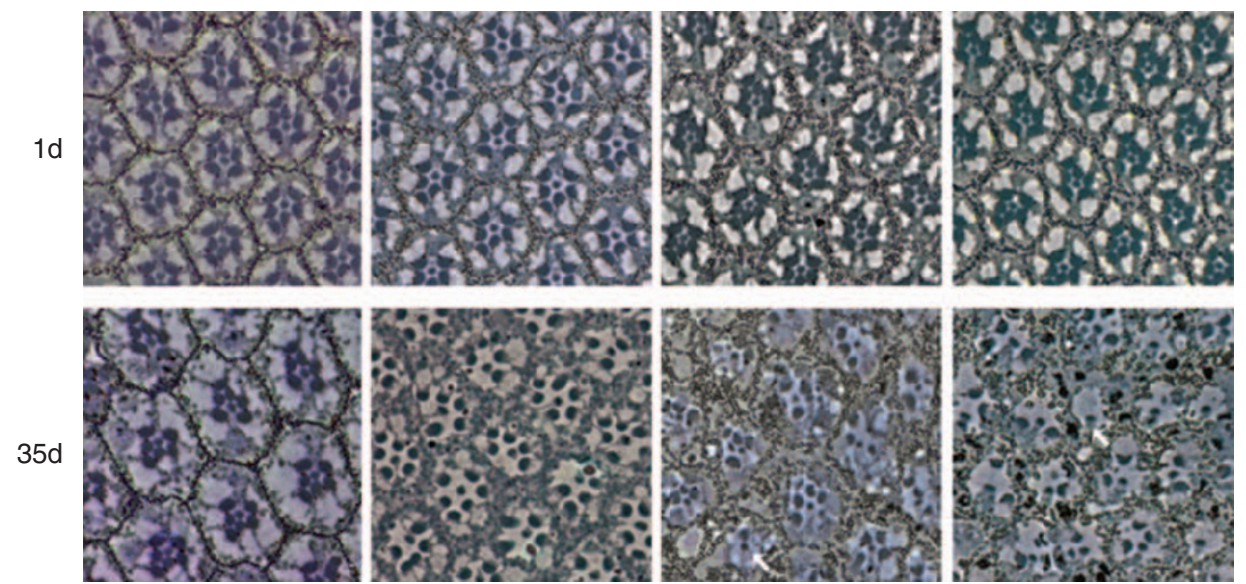

Figure 1 Neuronal degeneration by polyglutamine Atrophins. Ageing shown in all figures was performed at $29^{\circ} \mathrm{C}$. (a) A schematic representations of the fly Atro and human Atrophin-1 protein architecture and polyglutamine expansions. (b) Eye pictures of 1-month-old flies expressing different forms of Drosophila Atro and of human Atrophins with GMR-Gal4. Eye depigmentation is visible especially in GMR $>$ Atro75QNand in GMR $>$ At-1-65Q $\triangle \mathrm{C}$. (c) Tangential eye sections at the dorso-ventral midline of flies expressing different forms of Atro with Rh1-Gal4. Photoreceptor loss is observed in all Atro forms after 35 days, which is more severe with Atro66QC and Atro75QN (see Supplementary Figure 1 for quantification). The white arrows indicate intact R7 cells in ommatidia that have lost all or most other photoreceptors. The Rh1 driver is not expressed in R7/R8

expanded either stretch to engineer the Atro75QN and the Atro66QC proteins (Figure 1a and Supplementary Figure 1). The former carries a $75 \mathrm{Q}$ stretch in the middle of a polyprolin domain, in a position similar to where the Q stretch is found in human Atrophin-1 (At-1), whereas the latter carries a $66 \mathrm{Q}$ stretch at the $\mathrm{C}$-terminal end at a position where there is a $Q$ stretch not conserved with human At-1. Both Atro proteins are able to rescue Atro embryonic lethality, albeit with slightly different efficiency (Supplementary Table 1), indicating that polyQ expansion does not cause generalised loss of Atro function. Overexpression in the eye with GMR-Gal4 of Atro75QN or Atro66QC induces progressive depigmentation (Figure 1b), commonly observed in neurodegenerative models in the eye. In addition, expression of Atro only in the adult eye using the Rhodopsin1 (Rh1) driver is sufficient for degeneration, which is visible in terms of loss of photoreceptor cells specifically due to ageing (Figure 1c and Supplementary Figure 1). The latter experiment also shows that photoreceptor degeneration is strictly cell-autonomous as R7, the only cell that does not express Rh1-Gal4, is never lost (Figure 1c, arrows). With GMR-Gal4, it is possible to observe, in horizontal head sections, generalised tissue consumption, which leads to dramatic retinal collapse
(Figure 5, see also figure legend). This is specifically observed with ageing as all Atro-expressing flies display a correctly sized retina when they eclose from the pupal case (data not shown). In all these assays, Atro75QN results in a more severe phenotype despite lower full-length expression levels when compared with Atro wt and Atro 66QC (Supplementary Figure 1). Interestingly, Atro wt also induces statistically significant photoreceptor loss and retinal consumption, although at a much lower level than its polyQ counterparts (Figures $1 c$ and $5 b$ ). This raises the possibility that degeneration induced by polyQ Atro is a combination of polyQ toxicity and the detrimental effects of Atro itself.

As previously reported, ${ }^{16}$ and similar to fly Atro proteins, a human At-1 fragment with a polyQ expansion (At-1-65Q $\Delta \mathrm{C}$, which accumulates in DRPLA patients and has been shown to be an At-1 form of extreme toxicity ${ }^{17}$ ) induces progressive eye depigmentation (Figure 1b) and retinal collapse (Figure 5c) when expressed in the fly retina. Surprisingly expression of full-length human At-1 does not promote any major degeneration of the fly retina, also when carrying a $65 \mathrm{Q}$ tract (Figures $1 \mathrm{~b}$ and $5 \mathrm{c}$ ). Interestingly, full-length At-1, regardless of polyQ length, is expressed at low levels despite normal mRNA expression levels (Supplementary Figure 1), and this could explain the lack of a dramatic effect in the retina. The 
mechanism responsible for the low At-1 levels is unknown, but it does not involve protein degradation through the proteasome or through macroautophagy (Supplementary Figure 2). As the highly expressed $\Delta \mathrm{C}$ transgenes derive from the fulllength ones, differential translation efficiency is also unlikely, as they share the very same $5^{\prime}$ UTR and beginning of the ORF up to the point of deletion (aa 917).

Finally, intracellular aggregates, a hallmark of polyQ diseases, are formed upon polyQ Atrophin expression (Supplementary Figure 3). We have analysed in detail those formed by At-1-65Q $\Delta \mathrm{C}$ and found that this protein is able to recruit in its heavily ubiquitinated aggregates the endogenous fly Atro; general transcription factors such as the TATA-box Binding Protein (TBP); chaperones such as Hsp70; Nervy (the Drosophila orthologue of Atrophin-1 interactor ETO/MTG8 ${ }^{18}$ ) and chromatin-modifying enzymes such as the histone deacetylase Rpd3. However, the co-repressor Sin3A, often found in the same complex with Rpd3, and Mi-2, part of the NuRD chromatin remodelling complex, is not enriched in aggregates, indicating that these have a specific content rather than being a general protein trap.

Glial cells are particularly sensitive to polyglutamine Atrophins. Neurodegenerative phenotypes in the DRPLA fly models are not restricted to retinal photoreceptors. Neuronal overexpression of fly Atro results in developmental abnormalities ${ }^{19}$ that severely affect viability. However, switching on polyQ Atro only during adult life specifically affects fly longevity. Both Atro75QN and
Atro66QC are toxic when expressed in the glia (Figure 2a), albeit at different levels, whereas only Atro75QN is toxic in the neurons (Figure $2 \mathrm{~b}$ ). This suggests that glial cells are particularly sensitive to fly polyQ Atrophins. Also, glial expression does not affect development and the reduction in viability is primarily due to expression in adult life (Figure $2 \mathrm{a}$ and $\mathrm{c}$, and Supplementary Figure 4). Similarly, At-1$65 \mathrm{Q} \Delta \mathrm{C}$ but not its wt counterpart reduces fly viability if expressed in neurons or in the glia (Figure $2 e$ and $f$, and Supplementary Figure 4). Glial expression with two different drivers results in a stronger phenotype than in the case of neuronal expression. Interestingly, also, the low expressed At-1-65Q significantly reduces fly viability when driven in the glia but not in the neurons (Figure $2 e$ and $f$ ). Under the same conditions, flies expressing a form of human Huntingtin, $\mathrm{Htt}-$ exon-1-93Q, ${ }^{20}$ show on the contrary faster mortality upon neuronal expression (Supplementary Figure 4). Thus, our viability assays indicate that polyQ Atrophins are toxic both in neurons and glial cells, with a noticeable sensibility of glial cells specific to polyQ Atrophins.

In conclusion, all DRPLA fly models recapitulate typical polyQ toxicity but also suggest that protein-specific effects may modulate the phenotypes in comparison with other polyQ models.

Accumulation of autophagic hallmarks in degenerating neurons and glia. It has been reported that part of the toxicity exerted by polyQ proteins results from a block in the proteasome. ${ }^{21,22}$ However, neither Drosophila nor human a

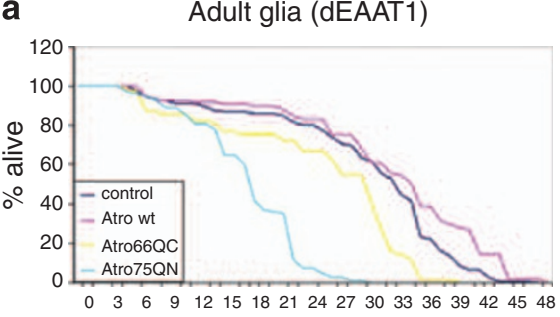

days

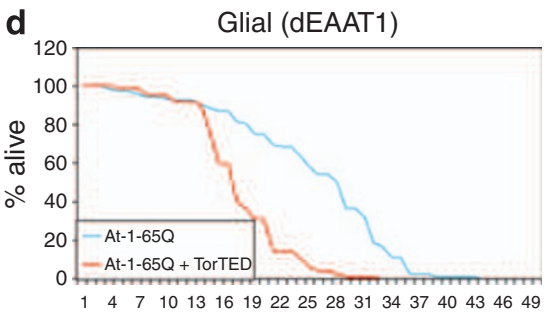

days b

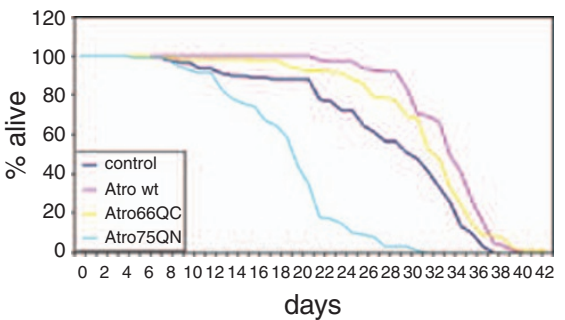

e

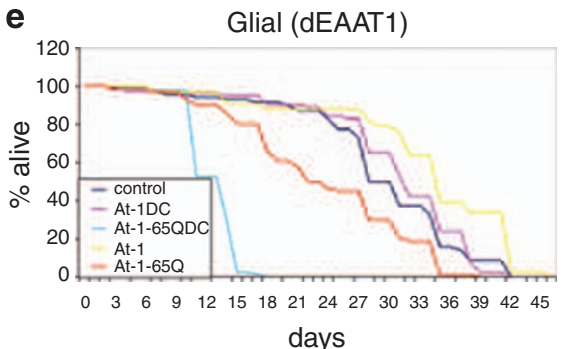

C

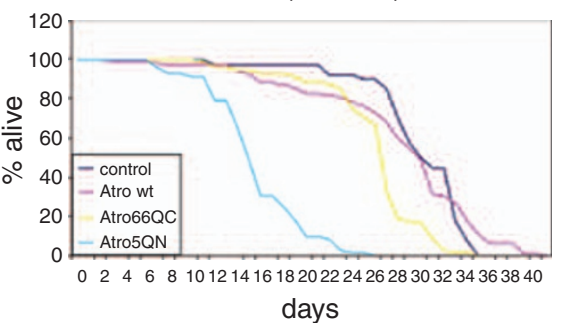

f

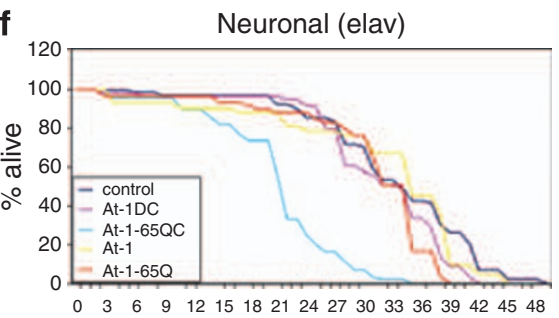

days

Figure 2 Glial and neuronal toxicity of Atrophins. (a) Viability curves for flies expressing specifically in the adult life different forms of Drosophila Atro in the glia with the dEAAT1 driver. Expression in adults has been achieved thanks to the Gal80 repressor carrying a temperature-sensitive mutation. (b) Viability curves for flies expressing specifically in the adult life different forms of Drosophila Atro in neurons with the Elav driver. (c) Viability curve of flies expressing different Drosophila Atro proteins in the glia. Atro75QN strongly reduces viability, whereas Atro66QC only causes a modest but significant (Kaplan-Meyer log rank 30.95; $P<0.0001$ ) reduction with respect to control flies. (d) Viability curves of flies expressing At-1-65Q in the glia with the $d E A A T 1$ driver either alone or in combination with Tor $^{\mathrm{TED}}$. Tor ${ }^{\mathrm{TED}}$ brings about a significant enhancement of fly mortality (Kaplan-Meyer log rank 93.42; $P<0.0001$ ). (e) Viability curve of flies expressing different human At-1 forms in the glia with the $d E A A T 1$ driver. At-1-65Q $\Delta C$ strongly reduces viability with respect to control flies, At-1-65Q causes a modest but significant reduction (Kaplan-Mayer log rank 16.52; $P<0.0001$ ) and full-length At-1 improves viability. (f) Viability curves for flies expressing different human At-1 forms in neurons with the Elav driver. Only At-1-65Q $\Delta \mathrm{C}$ reduces viability significantly with respect to control flies 
polyQ Atrophins impair proteasome functions extensively (Supplementary Figure 5). This suggests that although proteasomal impairment may contribute to their phenotype, their toxic effect is unlikely to be due to a massive block of the proteasome.

Overexpression of Atro through mutations in the microRNA mir8 has been shown to promote apoptosis during larval neuronal development. ${ }^{19}$ In aged adult retinae, significant accumulation of TUNEL signal is detected only if polyQ Atrophins are expressed in all eye cells with the GMR driver but not when the $R h 1$ driver is used to express the Atro proteins specifically in photoreceptor neurons (Supplementary Figure 5). Although these data confirm that polyQ Atrophins can stimulate apoptosis in developing tissues and non-neuronal adult eye cells, apoptosis does not appear to be chiefly responsible for the degeneration of adult post-mitotic photoreceptor neurons. TUNEL signal, in fact, correlates well with depigmentation, which could be due to apoptotic death of pigment cells.
At a finer level of analysis, electron microscopy (EM) of degenerating photoreceptors shows that wt Atro promotes the formation of autophagic vacuoles, which are rarely found in control retinae (Figure 3a). PolyQ Atrophins induce the formation of innumerable typical autophagosomes that cluster together and fuse with electron-dense lysosomal compartments to form large autophagolysosomes (Figure 3a). Accumulation of similar autophagic organelles is also found in glial cells expressing polyQ Atrophins just before organism death (Supplementary Figure 6). In addition, many mitochondria show abnormal morphology, although they are hardly present in strongly degenerated tissue (Figure 3a). The presence of autophagic organelles is confirmed for Atro66QC and Atro75QN by punctuated staining in the retina for GFP:: Atg8a, a marker for autophagy (Figure 3b). ${ }^{23}$ GFP ::Atg8a clustering in Atro wt is present but less substantial, in agreement with the electron microscopic analysis (Figure 3b).

In conclusion, autophagosomes and autophagic markers dramatically accumulate inside cells expressing polyQ Atrophins, a

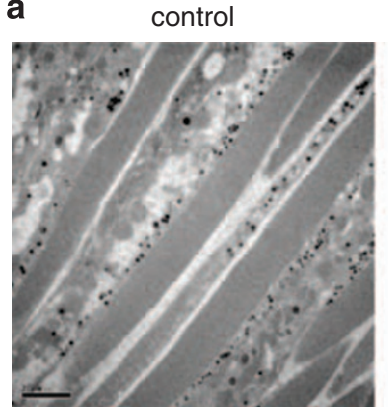

Atro66QC
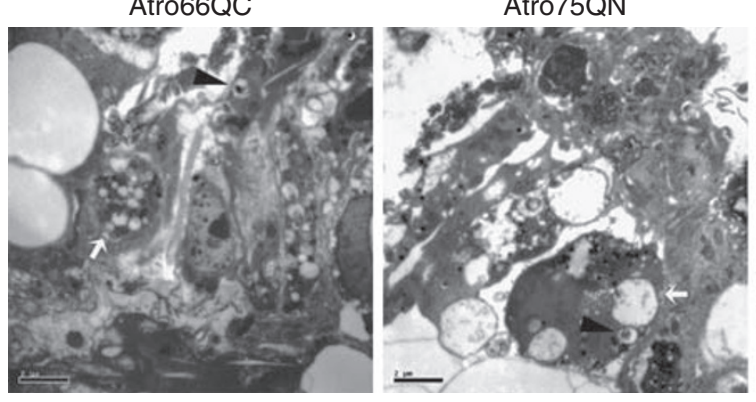

Atro75QN
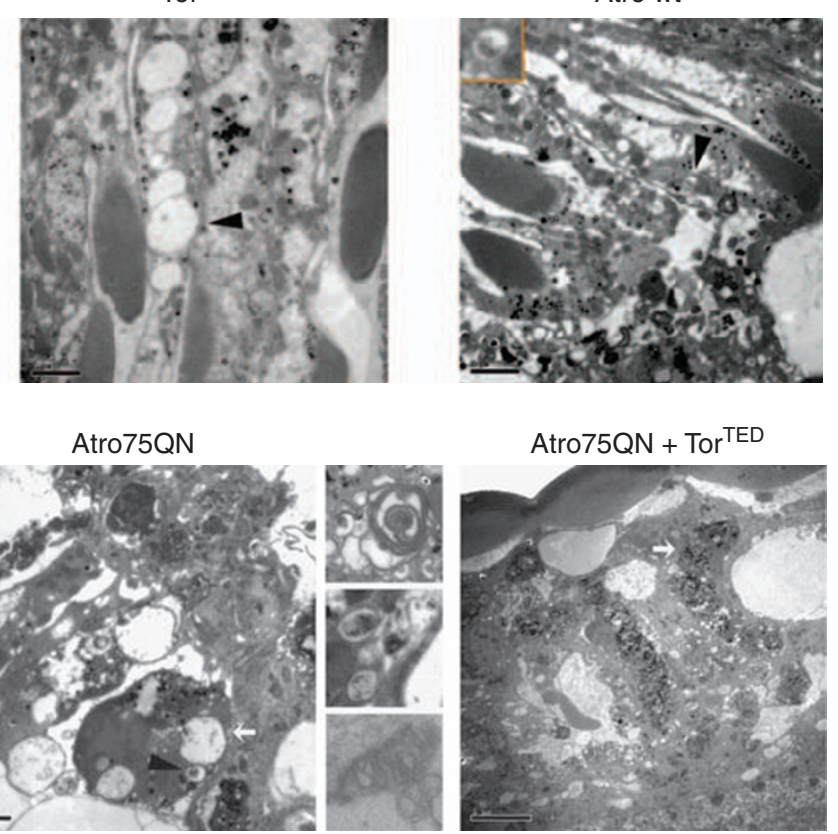

b.
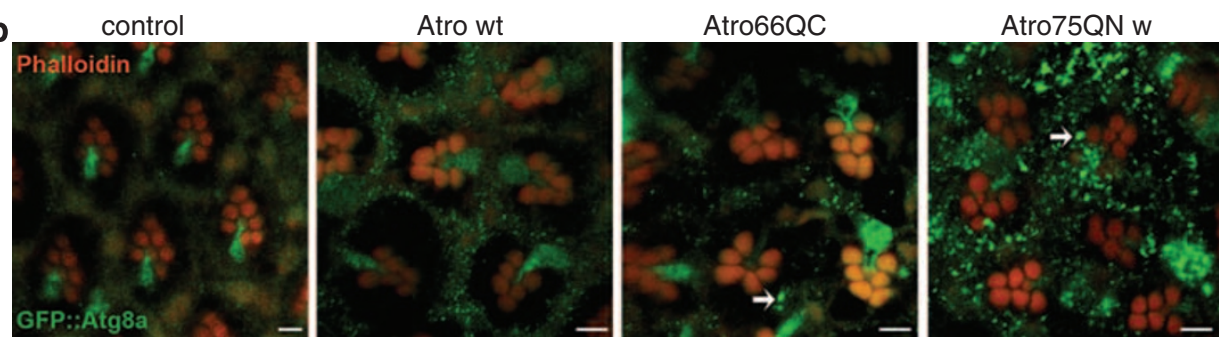

Figure 3 Autophagy in the DRPLA fly models. (a) Electron microscopic retinal pictures of control flies or flies expressing different Atro forms and/or Tor ${ }^{\text {TED }}$ with the GMR driver and aged 14 days. The arrowheads indicate autophagosomes (also shown in the top left zoom inset in the Atro wt panel), and the arrows indicate large autophagolysosomes that become gigantic with Tor ${ }^{\text {TED }}$. Control flies show virtually intact tissue, whereas expression of Tor ${ }^{\text {TED }}$ brings about the formation of large vacuoles that appear empty (arrowhead). Zoom in panels show (top to bottom) a multilamellar body, autophagosomes with undigested material and a damaged mitochondrion. Scale bars $2 \mu \mathrm{m}$, except Atro75QN + Tor ${ }^{\mathrm{TED}} 5 \mu \mathrm{m}$. (b) Confocal pictures of whole-mount retinae of flies expressing with GMR different Atro forms and GFP:: Atg8a, aged 10 days. Red indicates phalloidin-marking rhabdomeres, and green indicates GFP. Accumulation of small GFP::Atg8a dots is mostly in non-neuronal interommatidial cells with wt Atro. Atro66QC and Atro75QN cause the GFP signal to also gather in large masses inside the photoreceptors (arrows). R7 photoreceptors are marked unspecifically for a technical artefact. Scale bars $2 \mu \mathrm{m}$ 
suggesting a major role for autophagy in this form of cellular degeneration.

\section{Neurodegeneration is not rescued by further induction} of autophagy. Having established the presence of autophagy, we further analysed its functional significance for neurodegeneration in the DRPLA flies.

Blocking the signal responsible for autophagy induction ${ }^{23}$ by generating mutant clones for $\operatorname{atg} 1^{43 D}$ (a deletion of $966 \mathrm{bp}$ that includes the Atg1 translation start site ${ }^{23}$ and thereby a putative null allele) leads to an increase in the degeneration caused by polyQ Atro (Figure 4a), showing that endogenous autophagy attempts at protecting from neurodegeneration, as reported in other models. This is confirmed by enhancement of Atro retinal degeneration after RNAi-mediated downregulation $^{23}$ of Atg5, a principal autophagy factor (Figure 5b). Atg $5^{I R}$ also enhances fly mortality due to glial expression of polyQ Atrophins (Supplementary Figure 7). On the contrary, overexpressing the chaperone $\mathrm{dHdj} 1$ significantly suppresses polyQ Atro toxicity in both assays (Figure $5 \mathrm{~b}$ and data not shown). This indicates that it is possible to rescue the DRPLA flies through another mechanism known to protect from polyQ toxicity and argues for the specificity of the effect observed with atg $5^{I R}$.

Autophagy is controlled by the Tor-signalling pathway in response to several stimuli, ${ }^{3}$ and induction of further autophagy through inhibition of the Tor pathway has been reported to alleviate a number of neurodegenerative conditions. ${ }^{24-27}$ However, in our model, expression of a dominantnegative form of the Tor kinase, Tor ${ }^{T E D}$, a strong inducer of autophagy, ${ }^{23}$ enhances retinal degeneration by all Atro forms (Figure $5 \mathrm{~b}$ ). In addition, Tor ${ }^{\mathrm{TED}}$ expression further reduces the vitality of flies expressing At-1-65Q in the glia (Figure 2d) and results in lethality at larval stages when coexpressed in the glia with At-1-65Q $\Delta \mathrm{C}$ and any Drosophila Atro form (data not shown). On the contrary, Tor ${ }^{\mathrm{TED}}$ has no effect per se on viability and longevity if expressed in the glia under the same conditions (Supplementary Figure 7).

Likewise, addition of $1 \mu \mathrm{M}$ rapamycin, a potent inhibitor of Tor, in the fly food leads to significant and highly reproducible decrease in the number of photoreceptor cells per ommatidium in flies expressing Atro75QN (Figure 4b and Supplementary Table 2). Fly development was delayed by approximately 3 days on rapamycin treatment as previously described, ${ }^{23}$ indicating that the drug is effective under the conditions we used. Flies expressing human Htt-exon-1-93Q treated in parallel under the same conditions show small rescue (Supplementary Figure 7). Finally, rapamycin also enhances the mortality of flies expressing Atro75QN in the glia with the repo driver and heterozygous for the null $\operatorname{Tor}^{\Delta P}$ mutation (Supplementary Figure 7).

More direct stimulation of autophagy downstream from Tor, by overexpression of the atg1 gene, brings about an even more dramatic enhancement of retinal degeneration by all
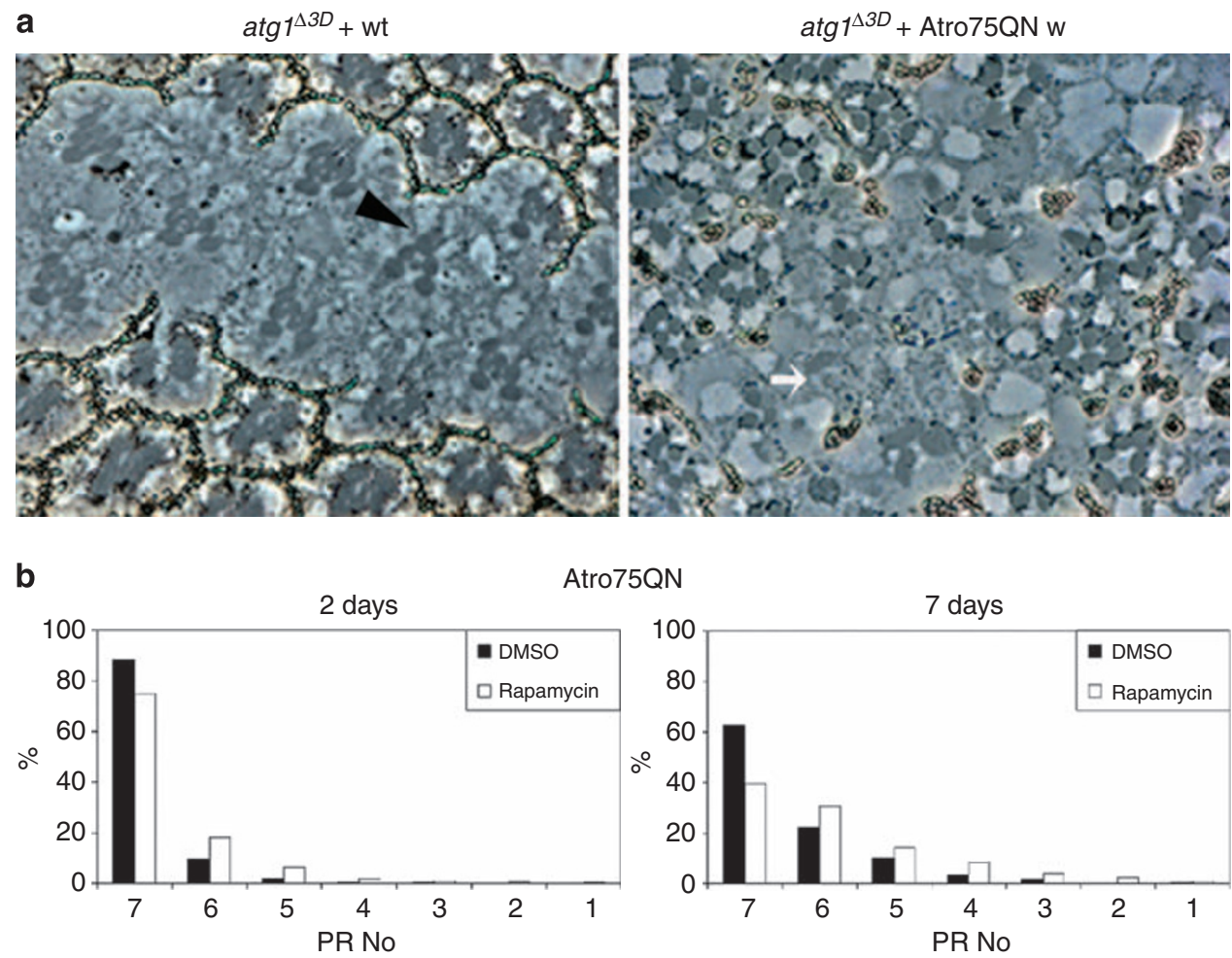

Figure 4 Modulation of autophagy does not rescue cell degeneration. (a) Tangential eye sections through $\operatorname{atg} 1^{43 D}$ clones (marked by the absence of yellow pigment) in flies either wt or expressing Atro75QN with GMR and aged 14 days. Many ommatidia show a severely reduced complement of photoreceptors specifically inside clones in Atro75QN-expressing flies (arrow). Only occasional photoreceptor loss is present inside clones in the wt background (arrowhead). (b) Histograms showing the number of photoreceptors (PR) of GMR $>$ Atro75QN/Tor ${ }^{P}$ flies on $1 \mu \mathrm{M}$ rapamycin food aged for 2 or 7 days. The 2-day chart represents masked counts of 1681 ommatidia (24 flies) for DMSO controls and 2514 ommatidia (34 flies) for rapamycin from three independent experiments $\left(\chi^{2}\right.$-value is $36.5 ; P<0.001$ for 6 degrees of freedom). The chart at 7 days represents 679 ommatidia (10 flies) for DMSO and 608 ommatidia (9 flies) for rapamycin from two independent experiments $\left(\chi^{2}\right.$-value $\left.77.9 ; P<0.001\right)$ 
a
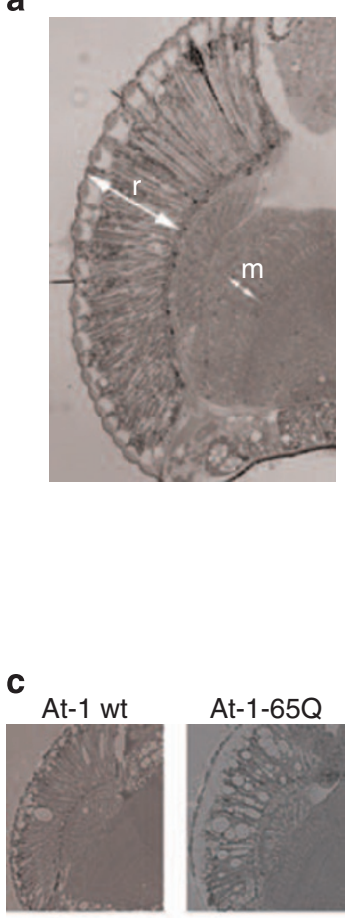

$3.81 \pm 0.17$

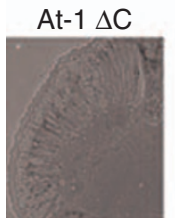

$3.64 \pm 0.11$

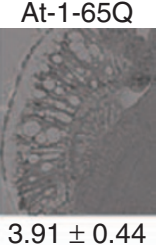

At-1-65Q $\Delta C$

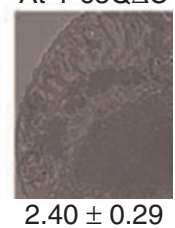

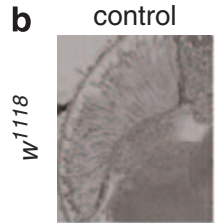

$3.75 \pm 0.50$

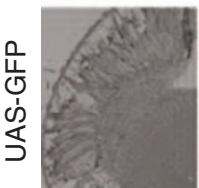

$3.62 \pm 0.21$

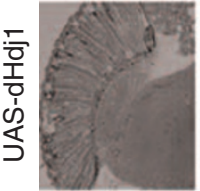

$3.50 \pm 0.22$

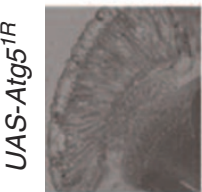

$3.51 \pm 0.33$

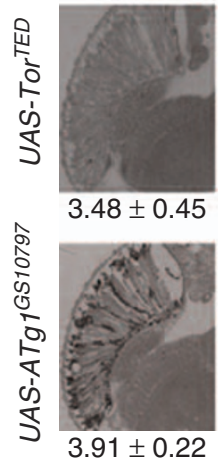

$3.91 \pm 0.22$
Atro wt

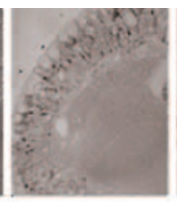

$2.32 \pm 0.33$
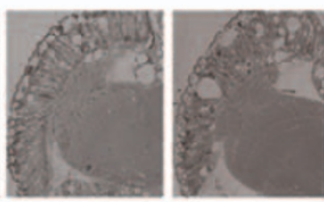

$2.21 \pm 0.34$
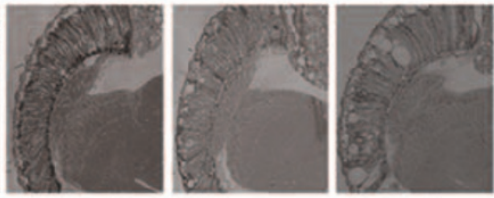

$2.62 \pm 0.24 \quad 2.36 \pm 0.32$
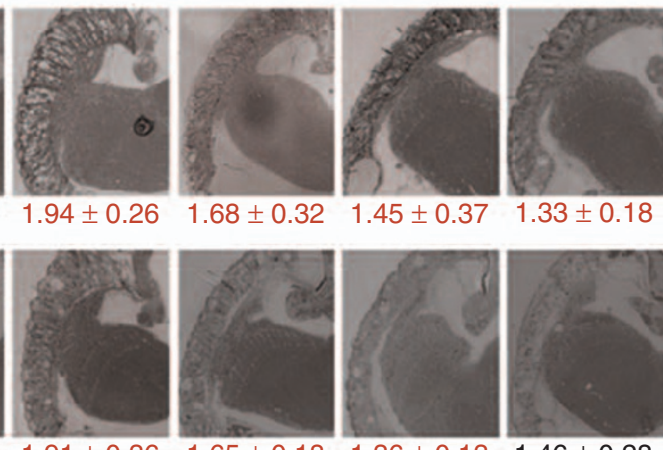

$1.33 \pm 0.18$
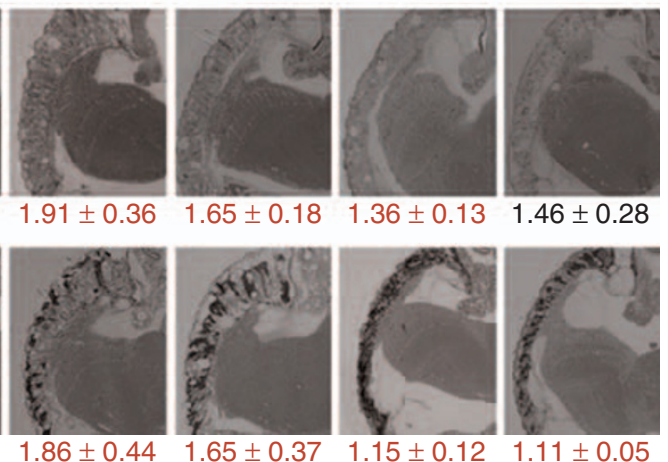

Figure 5 Genetic modifiers of retinal degeneration. (a) A horizontal head section of a wt fly at the dorso-ventral midline. The arrows indicate the extent of the retina ( $r$ ) and of the medulla $(\mathrm{m})$. (b) Horizontal head sections of flies overexpressing different forms of Drosophila Atro and/or other transgenes with the GMR driver. The sections are representative examples for each genotype and the ratio \pm standard deviation of the extent of the retina over the underlying medulla is reported in each panel. The medulla is unaffected by GMR expression and is a control for unspecific fly size effect. Flies were allowed to develop at $18^{\circ} \mathrm{C}$ and newly eclosed adults were aged at $29^{\circ} \mathrm{C}$ for 28 days. On average, six female heads coming from 2-3 independently aged populations were sectioned for each genotype. In the top row, all Atro proteins are being shown to cause a highly significant (two-tailed $t$-test; $P<0.001$ ) degeneration of the retinal tissue as compared with control flies (GMR-Gal4, left most). For genetic interactions with other mutants, statistically significant changes (two-tailed $t$-test; $P<0.05$ ) in comparison with the $w^{1118}$ negative control (first line of the corresponding column) are indicated in red. (c) Horizontal head sections of flies overexpressing different forms of human Atrophins with GMR-Gal4. Only At-1-65Q $\Delta \mathrm{C}$ causes a significant decrease in the extent of the retina, whereas At-1-65Q does not show an effect in this assay, despite the same $65 \mathrm{Q}$ expansion

Atro forms (Figure $5 b)$. We have used for this assay a weak UAS-Atg1 stock, ${ }^{28}$ obtained by insertion of the Gene Search transposon GSV6 upstream of the atg1 gene, whose moderate expression does not affect retinal consumption per se (Figure $5 b$ ).

In conclusion, our data indicate that although endogenous autophagy has a crucial role in moderating polyQ Atrophin toxicity, further induction of autophagy does not rescue the neurodegeneration of our DRPLA fly models, differently from what has been reported for other Drosophila neurodegeneration models. ${ }^{24,27}$
Autophagic digestion is blocked at the lysosomal level. Vacuoles formed upon Atro overexpression appear to be characteristically filled with unstructured partially degraded debris. On the contrary, expression of Tor ${ }^{\mathrm{TED}}$ or Atg1 promotes the formation of autophagic vacuoles that appear empty (Figure $3 \mathrm{a}$ and Supplementary Figure 6). In addition, autophagosomes fused with the dark electrondense lysosomes still retain their inner membrane (Figure 3a), which is normally readily degraded. These structures form when complete digestion of autophagolysosomes is being blocked with lysosomotropic 
agents. ${ }^{29}$ These data therefore suggest that Atro is partially blocking or abnormally delaying autophagy at the level of digestion of autophagosomes.

One possibility to explain the lack of digestion is that autophagosomes are not able to fuse correctly with lysosomes. However, in dissected single ommatidia in culture, many GFP::Atg8 punctae, which accumulate upon expression of Atro wt and more strongly upon Atro75QN expression, colocalize with lysotracker-positive compartments
(Figure 6a), indicating that fusion between autophagosomes and lysosomes is functional in these cells and that an eventual block of the autophagic flux is subsequent to the formation of autophagolysosomes. Also, lysotracker staining indicates correct acidification of autophagolysosomes. In fact, expression of all forms of Atro elicits massive accumulation of large acidic compartments, positive for lysotracker staining both in adult ommatidia and in imaginal discs (Figure 6a and Supplementary Figure 8). The small bright dots visible in the a

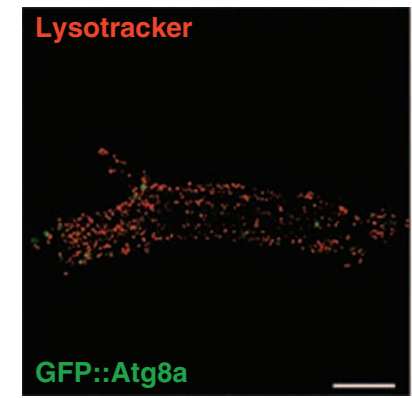

b

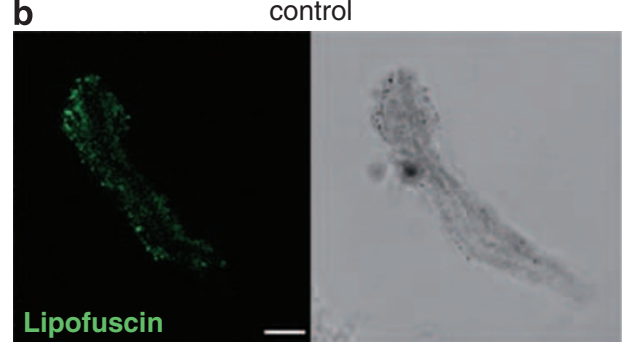

Atro wt

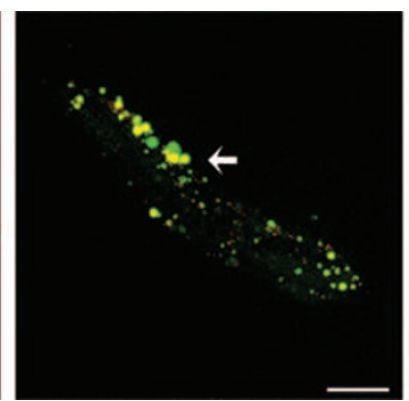

Atro75QN w

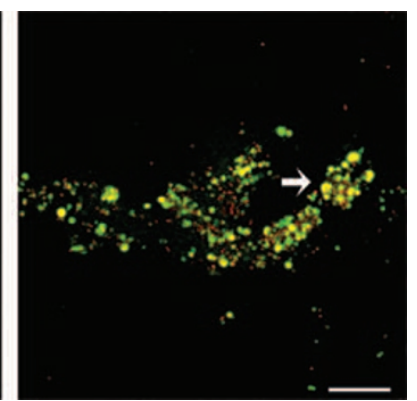

Atro75QN w

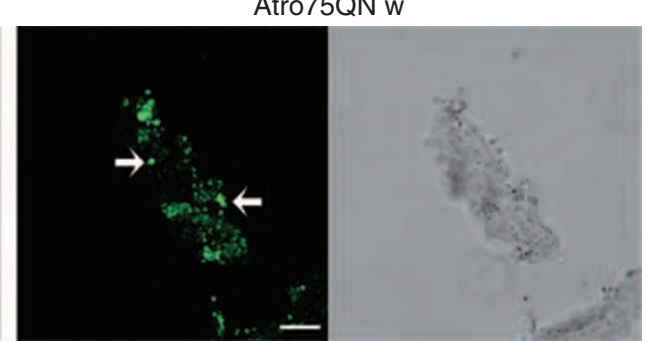

c

control

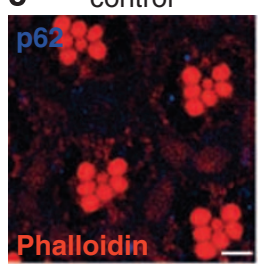

Atro66QC

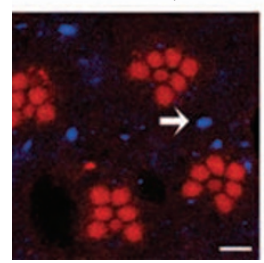

Atro wt

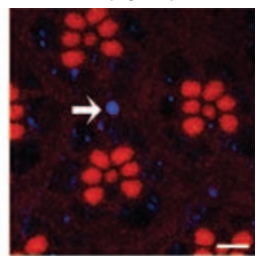

Atro75QN w

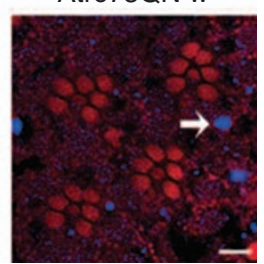

d Bulk Protein Degradation

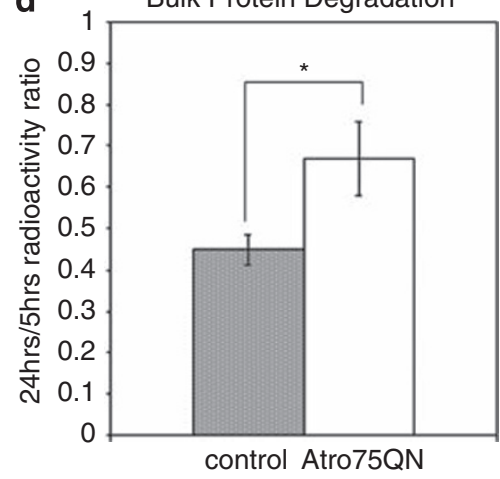

Figure 6 Lysosomal impairment by Atrophins. (a) Single ommatidia dissected from flies expressing GFP::Atg8 with GMR-Gal4 alone or in the presence of Atro wt or Atro75QN, aged 14 days. GFP fluorescence is indicated in green, and Lysotracker to mark acid lysosomes is indicated in red. The arrows indicate some of the many autophagolysosomes correctly formed, which appear yellow. Note that the small bright red dots are pigment granules (see Supplementary Figure 8). Scale bars $2 \mu \mathrm{m}$. (b) Single ommatidia dissected from control flies or flies expressing Atro75QN with GMR-Gal4, aged 14 days. The arrows indicate large vesicles of the autofluorescent lipofuscin pigment detected at $488 \mathrm{~nm}$. Small dots also found in control ommatidia are pigment granules. Scale bars $2 \mu \mathrm{m}$. (c) Confocal pictures of whole-mount retinae of flies expressing with GMR different Atro forms, aged 10 days. Red indicates phalloidin-marking rhabdomeres, and blue indicates p62. Atro wt and, more prominently, Atro66QC and Atro75QN cause accumulation of p62 in large masses (arrows). Scale bars $2 \mu \mathrm{m}$. (d) Histograms showing the ratio between the ${ }^{35} \mathrm{~S}$ radioactivity signal incorporated in the acid-insoluble proteins after a 24 -h chase with respect to $5 \mathrm{~h}$ of chase. BG3 neuronal cells were co-transfected with either a control pUAST plasmid or with pUAST-Atro75QN together with pMT-Gal4. Short-lived proteins, mainly degraded by the proteasome, do not contribute to the signal as they are eliminated before the 5 - $h$ chase. The quantified signal has also been standardised for any radioactive proteins present in the chase medium, which may contribute to the signal but escape intracellular degradation; * indicates $P<0.05$ in two-tailed $t$-test 
red lyostracker channel also in control ommatidia, however, are an artefact due to pigment granules that remain attached to dissected photoreceptors (Supplementary Figure 8). These data suggest increased lysosomal storage as a result of an Atrophin-specific effect. Indeed, autofluorescent ageing pigments such as lipofuscin, a product of unsaturated fatty acid oxidation associated with several lysosomal pathologies, that can be visualised by autofluorescence at $488 \mathrm{~nm},{ }^{30}$ accumulate in large vesicles in dissected single ommatidia that express Atro75QN (Figure 6b). Likewise, the Drosophila polyubiquitin-binding protein $\operatorname{Ref}(2) \mathrm{P} / \mathrm{p} 62,{ }^{31}$ a multifunctional scaffold protein that marks ubiquitinated protein aggregates destined to degradation through the autophagic-lysosomal machinery, ${ }^{32}$ accumulates in a number of small and large bodies upon wt Atro and, more conspicuously, upon polyQ Atro overexpression (Figure 6c).

To measure lysosomal activity as a whole, we have established a biochemically amenable system on the basis of cultured Drosophila BG3 cells, a neuronal line derived by larval CNS, which can be transiently transfected with an efficiency of $\sim 20 \%$ (Supplementary Figure 9 ). In addition, these cells already express Atro endogenously (Supplementary Figure 9). Therefore, in consideration of all these properties, BG3 cells constitute an ideal setup to complement and extend our in vivo observations in an in vitro system. Exogenous Atro mutants were overexpressed by using the Gal4/UAS system with the same vectors (pUAST-based) that we used to make our DRPLA transgenic flies and a $\mathrm{CuSO}_{4}$ inducible promoter present in the pMT-Gal4 plasmid (Supplementary Figure 9).

In this neuronal cell culture, we have measured bulk protein degradation upon radioactive labelling. This is the best established assay for monitoring, independently of any substrate and of autophagy, the function of lysosomes in degrading long-lived $\left(>4 \mathrm{~h}\right.$ half-life) proteins. $^{33}$ Cells transfected with Atro75QN are significantly impaired or slower than control-transfected cells in degrading, after $24 \mathrm{~h}$ of chase, the radioactivity present at $5 \mathrm{~h}$ of chase in trichloroacetic acid (TCA)-insoluble protein pellets (Figure 6d). These data, taking into account the transfection efficiency, indicate a strong deficiency in the degradation of long-lived proteins by the lysosomes upon expression of polyQ Atro.

Finally, in agreement with the hypothesis of lysosomal blockage, coexpression of Tor ${ }^{\text {TED }}$ or Atg1 with Atro75QN in the fly retina generates gigantic electron-dense autophagolysosomes with little sign of content digestion (Figure 3a and Supplementary Figure 6), which may explain the lack of rescue by additional induction of autophagy.

\section{Discussion}

In conclusion, our study suggests that neurodegeneration in the DRPLA flies results from partial inhibition or delay of autophagic digestion that shares many similarities with lysosomal storage disorders. ${ }^{30,34}$ We have generated new animal models for DRPLA in D. melanogaster. The DRPLA mouse models generated in the past ${ }^{35,36}$ have been shown to recapitulate much of the human pathology but they are less flexible and genetically amenable than Drosophila, which has proved to be a valuable tool for dissecting the polyQ pathology. ${ }^{8}$ Our new fly models will allow faster analysis of several aspects of DRPLA neurodegeneration, also allowing detailed comparison with other polyQ disease models. Thanks to the high structural and functional conservation in the Atrophin family, our observations are likely to be relevant to the human disease. In this report, we focus on the cellular mechanism of degeneration and highlight the role of autophagy and the specific deregulations due to polyQ Atrophins.

On expression of polyQ Atrophins, autophagosomes and their markers are readily detected in neuronal and glial cells. PolyQ proteins have been described to strongly induce autophagy, ${ }^{24}$ and this may explain the markedly increased presence of autophagic organelles upon polyQ Atrophin expression in comparison with wt Atro. However, this phenotype may result as well from a block in clearance, ${ }^{34}$ making it impossible to establish the actual rate of autophagosome formation. Indeed, we report that Atrophin expression impairs lysosomal degradation but not fusion between autophagosomes and lysosomes or correct acidification of autophagolysosomes. In photoreceptor neurons that express Atrophin mutants, there is an increase in lipidic lysosomal storage as detected by the autofluorescent lipofuscin pigment and abnormal accumulation of poly-ubiquitinated proteins destined to be degraded by the autophagy-lysosome system, as detected by p62. Finally, neuronal Drosophila cells transfected with polyQ Atro are defective in degradation of the lysosomally targeted long-lived proteins.

A similar accumulation of correctly acidified, but not degradative, autophagolysosomes has also been reported to impair autophagic flux in mammalian cells as a result of incorrect maturation of the autophagolysosomes. ${ }^{37}$ Further investigations will be required to identify the mechanism through which lysosomal digestion is impaired; however, we show that this is an intrinsic property of Atrophin, which may account for the degenerative effect of wt Atro and may significantly modulate the toxicity of polyQ Atrophins.

Autophagy has been reported to exert a protective function under a number of neurodegenerative conditions. ${ }^{24-27}$ Consistent with these reports, we find that blocking endogenous autophagy induction enhances neurodegeneration by Atrophins, as suggested by the atg1 clones. However, we have been unable to rescue Atrophin-mediated neurodegeneration through pharmacological or genetic induction of further autophagy. This is unique to the DRPLA flies in comparison with several other models of proteinopathies. At the ultrastructural level, further autophagy in the presence of Atrophin results in the formation of gigantic autophagolysosomes with little sign of content digestion. This provides an explanation for the lack of rescue as the full autophagy cycle cannot be completed.

However, we find that blocking autophagy is also detrimental to cells undergoing neurodegeneration by Atrophins. Thus, both blocking endogenous levels of autophagy and inducing further autophagy lead to stronger or faster degeneration. This apparent paradox can be explained either by implying that autophagy is not completely blocked by polyQ Atro or that the early events leading to autophagosome 
formation may themselves have a protective function. Also, in the absence of digestion, autophagosomes may indeed sequester potentially toxic material from the cytoplasm. We cannot however rigorously test these two hypotheses with the tools currently available.

Damaged mitochondria, which represent an important target of autophagy, may also contribute to cellular degeneration by Atrophins through impaired energy metabolism. Their disappearance, as well as that of most cytoplasmic content in blocked autophagic vesicles, may represent the final step in this form of autophagic neurodegeneration.

Autophagic degeneration by polyQ Atrophin is also induced in glial cells, where many of the observations made in photoreceptor neurons hold true. In addition to cell degeneration, expression of polyQ Atrophins in glial cells promotes pathology at the organismal level, leading to premature death, preceded by manifest locomotor deficits (data not shown). Toxicity upon glial expression is a common feature of several polyQ fly models ${ }^{38,39}$ and, interestingly, a recent report shows that in Huntington Drosophila models, glial cell degeneration affects fly viability, but through a different pathway than the one involved in brain neuronal toxicity. ${ }^{40}$ Remarkably, photorecteptors are affected by Huntingtin through the same pathway that affects the glia; however, glial cells appear to be more sensitive to expression of polyQ Atrophins in comparison with polyQ Huntingtin, which instead elicits a stronger effect when expressed in neurons.

The contribution of glial cells to the progression of neurodegeneration through the non-cell autonomous effects on neurons is an important subject of current investigations and it has been conclusively shown in the case of Amyotrophic Lateral Sclerosis (ALS) and Spino Cerebellar Ataxia 7 (SCA7). ${ }^{41,42}$ It is likely that glial cells are crucial also for most poly $Q$ diseases, given that poly $Q$ proteins are also expressed in the glia. In DRPLA, there is evidence that glial pathology is particularly important as intracellular aggregates have been detected in the glia and there is a significant correlation between glial cell death and degeneration of brain white matter. ${ }^{43,44}$

An important caveat of our DRPLA flies, which applies to all Drosophila models of polyQ diseases, is that they are based on overexpression of mutant proteins that are known to misfold as a consequence of polyQ expansion. Many of the described phenotypes could be a consequence of this artificial condition and our results will require validation in other model organisms, under expression of mutant Atrophins at endogenous levels. However, we report important differences with respect to other polyQ fly models, which are also based on the overexpression of misfolded polyQ proteins. This argues for the specificity of the degenerating mechanism described here. Thus, our new DRPLA fly models will be extremely useful to further dissect the role of the autophagic flux and of the glial contribution in neurodegeneration, and also as a means to explore new potential therapeutic approaches to DRPLA and related diseases.

\section{Materials and Methods}

Genetics. The following mutant fly stocks have been used: dEAAT1-Gal4, En-Gal4, GMR-Gal4, Rhodopsin1-Gal4, Tub-Gal4, Ptc-Gal4, Act5-Gal4, Elav-Gal4,
Repo-Gal4, UAS- ${ }_{N L S} L a c Z, \quad U A S-$ GFP $_{N L S}$, UAS-dHdj1, UAS-Atg5 ${ }^{1 R}$, UAS-

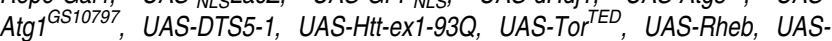

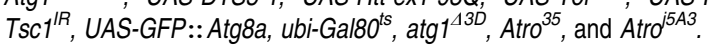

Rapamycin food. Fly-food was prepared according to our standard cornmeal recipe and DMSO or rapamycin (Sigma, St. Louis, MO, USA) dissolved $500 \mu \mathrm{g} / \mathrm{ml}$ in DMSO was added before preparing aliquots in 1:500 dilutions. Flies were crossed first on standard food at $18^{\circ} \mathrm{C}$, until early first instar larvae were visible. The adult population was then split in two and was allowed to lay eggs on either DMSO or rapamycin food at $18^{\circ} \mathrm{C}$. On pupal eclosion, $\mathrm{F} 1$ adults were transferred at $29^{\circ} \mathrm{C}$ on fresh DMSO or rapamycin food and flipped in a corresponding new vial every $48 \mathrm{~h}$. The embedded eyes of different genotypes were then sectioned and analysed in a blind manner and in one out of three independent experiments in a double blind manner.

Molecular cloning. DNA was cloned by standard techniques and by using a Gateway recombination system (Invitrogen, Carlsbad, CA, USA). Full-length or truncated atrophin-1 cDNAs encoding the first 917 amino acids (At-1- $\Delta \mathrm{C}$ or At-1-65Q $\Delta C$ ) were generated by PCR using full-length human atrophin-1 cDNAs (At-1 or At-1-65Q; a gift from David R Borchelt) as templates, and cloned into pUAST to generate UAS-At-1 $\triangle C$ and UAS-At-1-65QAC. To generate ubi-HA::At-1- $\triangle C$, an At-1 $\triangle C$ CDNA was cloned into the transformation vector pCaSpeR-UP-HA using the Gateway recombination system (Invitrogen).

For Drosophila Atro, a region containing the 65 CAG repeats of the At- 1 cDNA was amplified by PCR using oligos that insert an Spel site upstream from the CAG stretch and a BamHI site downstream from it. An Xbal-Bgll 1.4-kb fragment of Atro was mutagenised by PCR and a Gene Tailor (Invitrogen) to introduce an Spel and a BamHI site flanking the endogenous Q11 N-terminal stretch. On insertion of the 65 CAG, the Xbal-Bgll Atro fragment was reinserted into the pUAST-Atro plasmid to generate pUAST-Atro75QN. A 0.9-kb Notl fragment of Atro was mutagenised to insert the Spel and BamHI sites flanking the C-terminal region encoding for the Q14 stretch. Following insertion of the $65 \mathrm{CAGs}$, the Notl fragment was reinserted into the pUAST-Atro plasmid to generate pUAST-Atro66QC. Both Q stretches varied in length during cloning and two comparable stretches with similar expansion were chosen. All plasmids have been sequenced at MWG Biotech (Ebersberg, Germany). More detailed construct information is available on request.

RNA extraction and RT-PCR. RNA was extracted from 10-20 fly heads or BG3 cell pellets using an RNAgents kit (Promega, Madison, WI, USA); RNA quantity and quality were assessed using Nanodrop 1000 (Thermo Scientific, Waltham, MA, USA). A $0.5-\mu$ g weight of RNA sample was retro-transcribed using OdT or random primers and SuperScript III Reverse Transcriptase (Invitrogen) to generate cDNAs that were used as PCR templates. For qPCR, we used the SYBR Green JumpStart Taq ReadyMix (Sigma) and $500 \mathrm{nM}$ of specific primers for at-1 and rp49. Amplification and at-1 versus rp49 quantification was performed using an IQ5 (Bio-Rad, Hercules, CA, USA). Sequences of all the oligos are available on request.

Histology and EM. For retinal semi-thin sections, adult heads were treated and sectioned as previously described ${ }^{45}$ For electron microscopic sections, adult heads were dissected and fixed at RT in 4\% PFA and $2 \%$ glutaraldehyde in $0.12 \mathrm{M}$ sodium cacodylate buffer at $\mathrm{pH} 7.4$ for $1 \mathrm{~h}$. The heads were then washed $3 \times 10 \mathrm{~min}$ in $0.12 \mathrm{M}$ sodium cacodylate buffer, post-fixed in $2 \% \mathrm{OsO}_{4}$ in $0.12 \mathrm{M}$ sodium cacodylate buffer for $1 \mathrm{~h}$ and washed again $3 \times 10 \mathrm{~min}$. Afterwards, samples were dehydrated through an ethanol series and infiltrated with propylene oxide, embedded in epoxy resin (Fluka, Sigma) and polymerized at $80^{\circ} \mathrm{C}$. Ultrathin $(80 \mathrm{~nm})$ plastic sections were cut using a Leica UltraCut microtome using a diamond Diatome knife and post-stained with $2 \%$ uranyl acetate, followed by treatment with Reynolds' lead citrate, and stabilized for transmission EM by carbon coating. Examination was performed with a Zeiss Leo 912 microscope at $100 \mathrm{kV}$. Images were captured using a Gatan 792 Bioscan camera using Digital Micrograph as the software.

Immunohistochemistry. Larval imaginal discs and brains were treated as previously described. ${ }^{45}$

For Lysotracker staining, wing imaginal discs from third instar larvae were dissected in PBS and stained with LysoTracker Red DND-99 (Molecular Probes, Invitrogen) diluted 1: 1000 in PBS for $10 \mathrm{~s}$ after 3 min of fixation in 3\% formaldehyde (Polysciences Inc., Warrington, PA, USA). Mounting was performed in Vectashield and samples were immediately visualized by fluorescence microscopy. 
Immunostainings of adult retina were performed with whole mount preparation. Aged eyes were dissected and fixed in $4 \%$ formaldehyde in PBS for $1 \mathrm{~h}$. The brain was removed approximately $30 \mathrm{~min}$ into fixation. Fixed eyes were then given three 10-min washes in PBST (PBS plus 0.3\% Triton X-100). The washed eyes were incubated in primary antibody in PBST plus $5 \%$ goat serum overnight at $4{ }^{\circ} \mathrm{C}$. The eyes were given three 10-min washes in PBST and incubated for $4 \mathrm{~h}$ at RT in secondary antibody followed by overnight staining in $2 \mathrm{mg} / \mathrm{ml}$ rhodamine-conjugated phalloidin (Sigma). After incubation, the eyes were given three 10-min PBST washes and mounted in Vectashield.

TUNEL staining of fixed imaginal discs and adult retinae was performed using the DeadEnd kit (Promega), according to the protocol from the supplier.

Single ommatidia were dissected following a protocol described by RC Hardie. ${ }^{46}$ Briefly, adult retinae were grossly dissected in Schneider's insect medium supplemented with $10 \%$ foetal calf serum (FCS). The dissected retina were then transferred in a drop of fresh new Schneider's medium with 10\% FCS on glass slides and ommatidia were dissected with fine forceps. At this point, LysoTracker Red DND-99 (Molecular Probes) was added if required, and then most of the medium was removed with a pipette before adding Vectashield and sealing with a coverslip. All samples were imaged within $24 \mathrm{~h}$.

The following antibodies were used: goat anti-human Atrophin-1 APG840 $(1 / 500),{ }^{18}$ rat anti-Elav $(1 / 10$; hybridoma supernatant 7E8A10 from DSHB, lowa City, IA, USA), mouse anti-Repo (1/10, 8D12; DSHB) rabbit anti- $\beta$-galactosidase (1/1000; Cappel ICN-Pharmaceuticals Inc., Costa Mesa, CA, USA), mouse anti-HA (1/1000,16B12; Covance, Princeton, NJ, USA), rabbit anti-GFP (1:500; Molecular Probes), rabbit anti-Atro (1/500), rabbit anti-p62 (1/2000; a gift from Didier Contamine), mouse anti-HSP70 (1/500, MA3-007; Affinity BioReagents, Rockford, IL, USA), mouse anti-ubiquitin (1/200, 13-1600; Zymed, San Francisco, CA, USA), mouse anti-TBP (1/200, sc-421; Santa Cruz Biotechnology, Santa Cruz, CA, USA), rabbit anti-Nervy (1/300; a gift from Richard S. Mann), rabbit anti-Sin3A (1/200) and rabbit anti-RPD3 (1/200;a gift from Lori Pile), and rabbit anti-Mi2 (1/500; a gift from Alexander Brehm) and mouse anti-PolyQ (1/200, IC2; Chemicon, Temecula, CA, USA ). The secondary fluorescence-conjugated antibodies from Molecular Probes or Jackson Laboratories (Bar Harbor, ME, USA) were used at $1: 200$ dilutions. The samples were viewed using Zeiss Axioplanll, Bio-Rad and Zeiss LSM confocal microscopes.

Western blots. For flies, 10 fly heads were dissected, mashed directly in Laemmli buffer and boiled at $95^{\circ} \mathrm{C}$ for $5 \mathrm{~min}$. For cells, a T25 flask was harvested, pelleted and resuspended in Laemmli buffer. Polyacrylamide gel electrophoresis, blotting and antibody staining were performed according to standard protocols. Amersham (GE Healthcare, Piscataway, NJ, USA) ECL reagents were used for chemiluminescence. The following primary antibodies were used: anti-human Atrophin-1 APG840 (1/500) and anti-Atro (1/200).

Cell cultures and metabolic labelling. Neuronal BG3 cells (clone-1) were obtained from the Drosophila Genomics Resource Center and cultured in flasks with Shields and Sang M3 Insect Medium (Sigma) supplemented with 10\% FCS (Gibco, Invitrogen), PenStrep and insulin. For the highest transfection efficiency ( $\sim 20 \%)$, we used the Effectene kit (Qiagen, Hilden, Germany).

For radioactive metabolic labelling, cells at $50-70 \%$ confluence were transiently transfected with Endofree-purified (Qiagen), copper-inducible pMT-Gal4 and a molar excess (two-fold) of UAS vectors (pUAST or pUAST-Atro75QN). After $24 \mathrm{~h}$ incubation in the transfection medium, each flask containing cells was incubated for $48 \mathrm{~h}$ in a radioactive medium containing $12.5 \mathrm{MBq}$ of EasyTag EXPRE ${ }^{35} \mathrm{~S}^{35} \mathrm{~S}$ mix (Perkin Elmer, Waltham, MA, USA) and $1 \mathrm{mM} \mathrm{CuSO}_{4}$. Thereafter, cells were incubated for 5 or $24 \mathrm{~h}$ with a chase medium containing $1 \mathrm{mM} \mathrm{CuSO}_{4}$, then harvested and lysed in PBS $+1 \%$ Triton X-100. Proteins were precipitated with $10 \%$ TCA and resuspended in Laemmli buffer. The samples were loaded onto a $10 \%$ SDS-PAGE gel, and the gel was dried and exposed to a phosphoimager screen. Signals were scanned using a Typhoon 8600 (Molecular Dynamics, GE Healthcare) and volumetrically quantified using the ImageQuant software. The radioactivity for each TCA pellet was standardised over the radioactivity present in the chase medium as secreted proteins that escape intracellular degradation. Finally, with these standardised values, the 24/5-h ratio was established for each transfection in each experiment according to the formula $r=\left(P_{24} \mathrm{~h} / \mathrm{M}_{24} \mathrm{~h}\right) /\left(\mathrm{P}_{5 \mathrm{~h}} / \mathrm{M}_{5 \mathrm{~h}}\right)$.

\section{Conflict of Interest}

The authors declare no conflict of interest.
Acknowledgements. We thank Gabriele Schilling, David Borchelt, Helen McNeill, Joe Bateman, Tor Erik Rusten, Serge Birman, Thomas Neufeld, the DHSB, DGRC and Bloomington for reagents and stocks; Julien Royet, Sharon Tooze and Ana Cuervo for discussions; Aicha Ouane for technical assistance; and Joy Burchell and Steve Catchpole for help with the phosphoimager. MF is supported by the Italian Telethon Foundation with a career development award and is an Assistant Telethon Scientist. This work was also supported by the European Union Marie Curie European Reintegration Grant 505739, by Fondazione Cariplo and Compagnia di San Paolo grants to MF, and funds from the Centre National de la Recherche Scientifique to $\mathrm{BC}$ and the $\mathrm{KCL}$ School of Biomedical and Health Sciences to MF.

1. Ross CA. Polyglutamine pathogenesis: emergence of unifying mechanisms for Huntington's disease and related disorders. Neuron 2002; 35: 819-822.

2. Arrasate M, Mitra S, Schweitzer ES, Segal MR, Finkbeiner S. Inclusion body formation reduces levels of mutant huntingtin and the risk of neuronal death. Nature 2004; 431 : 805-810.

3. Mizushima N, Levine B, Cuervo AM, Klionsky DJ. Autophagy fights disease through cellular self-digestion. Nature 2008; 451: 1069-1075.

4. Nixon RA. Autophagy in neurodegenerative disease: friend, foe or turncoat? Trends Neurosci 2006; 29: 528-535.

5. Chen HK, Fernandez-Funez P, Acevedo SF, Lam YC, Kaytor MD, Fernandez MH et al. Interaction of Akt-phosphorylated ataxin-1 with 14-3-3 mediates neurodegeneration in spinocerebellar ataxia type 1. Cell 2003; 113: 457-468.

6. Emamian ES, Kaytor MD, Duvick LA, Zu T, Tousey SK, Zoghbi HY et al. Serine 776 of ataxin-1 is critical for polyglutamine-induced disease in SCA1 transgenic mice. Neuron 2003; 38: 375-387.

7. Warrick JM, Morabito LM, Bilen J, Gordesky-Gold B, Faust LZ, Paulson HL et al. Ataxin-3 suppresses polyglutamine neurodegeneration in Drosophila by a ubiquitin-associated mechanism. Mol Cell 2005; 18: 37-48.

8. Marsh JL, Thompson LM. Drosophila in the study of neurodegenerative disease. Neuron 2006; 52: 169-178.

9. Romero E, Cha GH, Verstreken P, Ly CV, Hughes RE, Bellen HJ et al. Suppression of neurodegeneration and increased neurotransmission caused by expanded full-length huntingtin accumulating in the cytoplasm. Neuron 2008; 57: 27-40.

10. Naito $\mathrm{H}$, Oyanagi S. Familial myoclonus epilepsy and choreoathetosis: hereditary dentatorubral-pallidoluysian atrophy. Neurology 1982; 32: 798-807.

11. Nagafuchi S, Yanagisawa H, Ohsaki E, Shirayama T, Tadokoro K, Inoue T et al. Structure and expression of the gene responsible for the triplet repeat disorder, dentatorubral and pallidoluysian atrophy (DRPLA). Nature genetics 1994; 8: 177-182.

12. Erkner A, Roure A, Charroux B, Delaage M, Holway N, Core N et al. Grunge, related to human Atrophin-like proteins, has multiple functions in Drosophila development. Development 2002; 129: 1119-1129.

13. Shen Y, Lee G, Choe Y, Zoltewicz JS, Peterson AS. Functional architecture of atrophins. $J$ Biol Chem 2007; 282: 5037-5044.

14. Zhang S, Xu L, Lee J, Xu T. Drosophila atrophin homolog functions as a transcriptional corepressor in multiple developmental processes. Cell 2002; 108: 45-56.

15. Zoltewicz JS, Stewart NJ, Leung R, Peterson AS. Atrophin 2 recruits histone deacetylase and is required for the function of multiple signaling centers during mouse embryogenesis. Development 2004; 131: 3-14.

16. Charroux B, Freeman M, Kerridge S, Baonza A. Atrophin contributes to the negative regulation of epidermal growth factor receptor signaling in Drosophila. Dev Biol 2006; 291: 278-290.

17. Nucifora Jr FC, Ellerby LM, Wellington CL, Wood JD, Herring WJ, Sawa A et al. Nuclear localization of a non-caspase truncation product of atrophin-1, with an expanded polyglutamine repeat, increases cellular toxicity. J Biol Chem 2003; 278: 13047-13055.

18. Wood JD, Nucifora Jr FC, Duan K, Zhang C, Wang J, Kim Y et al. Atrophin-1, the dentatorubral and pallido-luysian atrophy gene product, interacts with ETO/MTG8 in the nuclear matrix and represses transcription. J Cell Biol 2000; 150: 939-948.

19. Karres JS, Hilgers V, Carrera I, Treisman J, Cohen SM. The conserved microRNA miR-8 tunes atrophin levels to prevent neurodegeneration in Drosophila. Cell 2007; 131: 136-145.

20. Steffan JS, Bodai L, Pallos J, Poelman M, McCampbell A, Apostol BL et al. Histone deacetylase inhibitors arrest polyglutamine-dependent neurodegeneration in Drosophila. Nature 2001; 413: 739-743.

21. Holmberg Cl, Staniszewski KE, Mensah KN, Matouschek A, Morimoto RI. Inefficient degradation of truncated polyglutamine proteins by the proteasome. EMBO J 2004; 23 . 4307-4318.

22. Venkatraman P, Wetzel R, Tanaka M, Nukina N, Goldberg AL. Eukaryotic proteasomes cannot digest polyglutamine sequences and release them during degradation of polyglutamine-containing proteins. Mol Cell 2004; 14: 95-104.

23. Scott RC, Schuldiner O, Neufeld TP. Role and regulation of starvation-induced autophagy in the Drosophila fat body. Dev Cell 2004; 7: 167-178.

24. Ravikumar B, Vacher C, Berger Z, Davies JE, Luo S, Oroz LG et al. Inhibition of mTOR induces autophagy and reduces toxicity of polyglutamine expansions in fly and mouse models of Huntington disease. Nat Genet 2004; 36: 585-595. 
25. Berger Z, Ravikumar B, Menzies FM, Oroz LG, Underwood BR, Pangalos MN et al Rapamycin alleviates toxicity of different aggregate-prone proteins. Hum Mol Genet 2006; 15: 433-442.

26. Sarkar S, Krishna G, Imarisio S, Saiki S, O'Kane CJ, Rubinsztein DC. A rational mechanism for combination treatment of Huntington's disease using lithium and rapamycin. Hum Mol Genet 2008; 17: 170-178.

27. Wang T, Lao U, Edgar BA. TOR-mediated autophagy regulates cell death in Drosophila neurodegenerative disease. J Cell Biol 2009; 186: 703-711.

28. Scott RC, Juhasz G, Neufeld TP. Direct induction of autophagy by Atg1 inhibits cell growth and induces apoptotic cell death. Curr Biol 2007; 17: 1-11.

29. Degtyarev M, De Maziere A, Orr C, Lin J, Lee BB, Tien JY et al. Akt inhibition promotes autophagy and sensitizes PTEN-null tumors to lysosomotropic agents. J Cell Biol 2008; 183: $101-116$.

30. Venkatachalam K, Long AA, Elsaesser R, Nikolaeva D, Broadie K, Montell C. Motor deficit in a Drosophila model of mucolipidosis type IV due to defective clearance of apoptotic cells. Cell 2008; 135: 838-851.

31. Nezis IP, Simonsen A, Sagona AP, Finley K, Gaumer S, Contamine D et al. Ref(2)P, the Drosophila melanogaster homologue of mammalian $\mathrm{p} 62$, is required for the formation of protein aggregates in adult brain. $\mathrm{J}$ Cell Biol 2008; 180: 1065-1071.

32. Bjorkoy G, Lamark T, Brech A, Outzen H, Perander M, Overvatn A et al. p62/SQSTM1 forms protein aggregates degraded by autophagy and has a protective effect on huntingtininduced cell death. J Cell Biol 2005; 171: 603-614.

33. Cuervo AM, Dice JF, Knecht E. A population of rat liver lysosomes responsible for the selective uptake and degradation of cytosolic proteins. J Biol Chem 1997; 272 5606-5615.

34. Settembre C, Fraldi A, Jahreiss L, Spampanato C, Venturi C, Medina D et al. A block of autophagy in lysosomal storage disorders. Hum Mol Genet 2008; 17: 119-129.

35. Sato T, Miura M, Yamada M, Yoshida T, Wood JD, Yazawa I et al. Severe neurological phenotypes of Q129 DRPLA transgenic mice serendipitously created by en masse expansion of CAG repeats in Q76 DRPLA mice. Hum Mol Genet 2009; 18: 723-736.
36. Schilling G, Wood JD, Duan $\mathrm{K}$, Slunt $\mathrm{HH}$, Gonzales V, Yamada M et al. Nuclear accumulation of truncated atrophin-1 fragments in a transgenic mouse model of DRPLA. Neuron 1999; 24: 275-286.

37. Razi M, Chan EY, Tooze SA. Early endosomes and endosomal coatomer are required for autophagy. J Cell Biol 2009; 185: 305-321.

38. Tamura T, Sone M, Yamashita M, Wanker EE, Okazawa H. Glial cell lineage expression of mutant ataxin-1 and huntingtin induces developmental and late-onset neuronal pathologies in Drosophila models. PLoS One 2009; 4: e4262.

39. Kretzschmar D, Tschape J, Bettencourt Da Cruz A, Asan E, Poeck B, Strauss R et al. Glia and neuronal expression of polyglutamine proteins induce behavioral changes and aggregate formation in Drosophila. Glia 2005; 49: 59-72.

40. Lievens JC, Iche M, Laval M, Faivre-Sarrailh C, Birman S. AKT-sensitive or insensitive pathways of toxicity in glial cells and neurons in Drosophila models of Huntington's disease. Hum Mol Genet 2008; 17: 882-894.

41. Custer SK, Garden GA, Gill N, Rueb U, Libby RT, Schultz C et al. Bergmann glia expression of polyglutamine-expanded ataxin-7 produces neurodegeneration by impairing glutamate transport. Nat Neurosci 2006; 9: 1302-1311.

42. Boillee S, Vande Velde C, Cleveland DW. ALS: a disease of motor neurons and their nonneuronal neighbors. Neuron 2006; 52: 39-59.

43. Hayashi $Y$, Kakita A, Yamada M, Koide R, Igarashi S, Takano $\mathrm{H}$ et al. Hereditary dentatorubral-pallidoluysian atrophy: detection of widespread ubiquitinated neuronal and glial intranuclear inclusions in the brain. Acta Neuropathologica 1998; 96 : 547-552.

44. Yamada M, Sato T, Tsuji S, Takahashi H. Oligodendrocytic polyglutamine pathology in dentatorubral-pallidoluysian atrophy. Ann Neurol 2002; 52: 670-674.

45. Montrasio S, Mlodzik M, Fanto M. A new allele uncovers the role of echinus in the control of ommatidial rotation in the Drosophila eye. Dev Dyn 2007; 236: $2936-2942$.

46. Hardie RC. Voltage-sensitive potassium channels in Drosophila photoreceptors. J Neurosci 1991; 11: 3079-3095.

\section{Supplementary Information accompanies the paper on Cell Death and Differentiation website (http://www.nature.com/cdd)}

